\title{
ON THE USE OF PERIODIC BOUNDARY CONDITIONS IN DISLOCATION DYNAMICS SIMULATIONS
}

\author{
Ronan Madec, Benoit Devincre* and Ladislas Kubin ${ }^{*}$ \\ DPTA, Commissariat à l'Energie Atomique, BP12, 91680 Bruyères-le-Châtel, France \\ *LEM, CNRS-ONERA, 29 Av. de la Division Leclerc, BP 72, 92322 Châtillon Cedex, France
}

\begin{abstract}
The use of periodic boundary simulations in dislocation dynamics simulations results in indesirable self-annihilation events. Methods are presented for avoiding this artefact and prescribing realistic dislocation mean free-paths.
\end{abstract}

Key words: dislocation dynamics simulation, periodic boundary condition.

\section{INTRODUCTION}

The present work is dealing with periodic boundary conditions (PBCs) in dislocation dynamics (DD) simulations. For the so-called mass simulations, PBCs are needed in order to ensure that dislocation fluxes are balanced at the boundaries of the primary simulation cell in order to avoid undesirable size effects due to finite dimensions and artificial dislocation losses due to image forces.

Bulatov, Rhee and $\mathrm{Cai}^{1}$ have discussed the application of PBCs to DD simulations. In short, dislocations glide in a periodic array formed by a primary simulation cell and its replicas. Every time a portion of dislocation line crosses a boundary between two cells, its images emerge in all the cells at the equivalent position on the opposite boundary. A balance of fluxes is then established through all the internal interfaces of the simulated volume. 
However, the application of PBCs to linear objects is known to lead to spurious self-interactions ${ }^{2}$. In the case of dislocations, a given portion of line may self-annihilate with one of its images after a certain number of boundary crossings. Self-annihilation may reduce the mean free-path of dislocations, and this can have drastic consequences that have not been considered so far in the context of DD simulations. A too short effective mean free-path affects the density of mobile dislocations and their storage rate and, hence, both the arrangement of the microstructure and the strain hardening properties.

The object of the present study is to discuss several methods that have been developed in order to control this artifact. These methods apply to DD simulations in which, like in real crystals, the spacing between slip planes obeys crystallographic relations ${ }^{3}$.

In part 2, a few definitions are given that are further used for calculating the self-annihilation distances. For particular slip geometries, self-annihilation occurs after a single boundary crossing and two methods are proposed for dealing with such situations. In part 3, it is shown that selfannihilation distances can be controlled by adequately tailoring the dimensions of orthorhombic simulation cells. Two model situations of practical interest are considered, those of isotropic and strongly anisotropic dislocation loops. Concluding remarks are presented in part 4.

\section{DIRECT SELF-ANNIHILATION}

\subsection{Self-Annihilation: Definitions}

Figure 1 shows a simple two-dimensional example of PBCs applied to a square cell of side $L$. Every dislocation line crossing the boundary of a simulation cell, enters all replicas at positions defined by translation vectors parallel to the cell sides, $\boldsymbol{L}_{\mathrm{x}}$ or $\boldsymbol{L}_{\mathrm{y}}$. These vectors are called modulo translations, as their linear combinations "fold" the microstructure developing in a slip plane into a set of images in every cell. This method has several advantages from a computational viewpoint ${ }^{1}$. It respects the continuity of the lines at the cell boundaries. In addition, shifting in an arbitrary manner the origin of the primary cell does not modify the abovementioned properties, so that there is actually no boundary to deal with in the presence of PBCs. Finally, any segment can be set at the center of a primary cell for the computation of its elastic interactions with the rest of the microstructure.

As the array of cells is periodic, its section by a slip plane is also periodic, albeit with a different period. A portion of dislocation line, then, necessarily meets with one of its images after a certain glide path. For instance in the square cell of Fig. 1- $a$ and for a portion of dislocation line propagating along 
the second diagonal, self-annihilation occurs after two boundary crossings only. The simplest way to control these undesirable spatial correlations consists in introducing orthorhombic distortions of the square or cubic simulation cells.
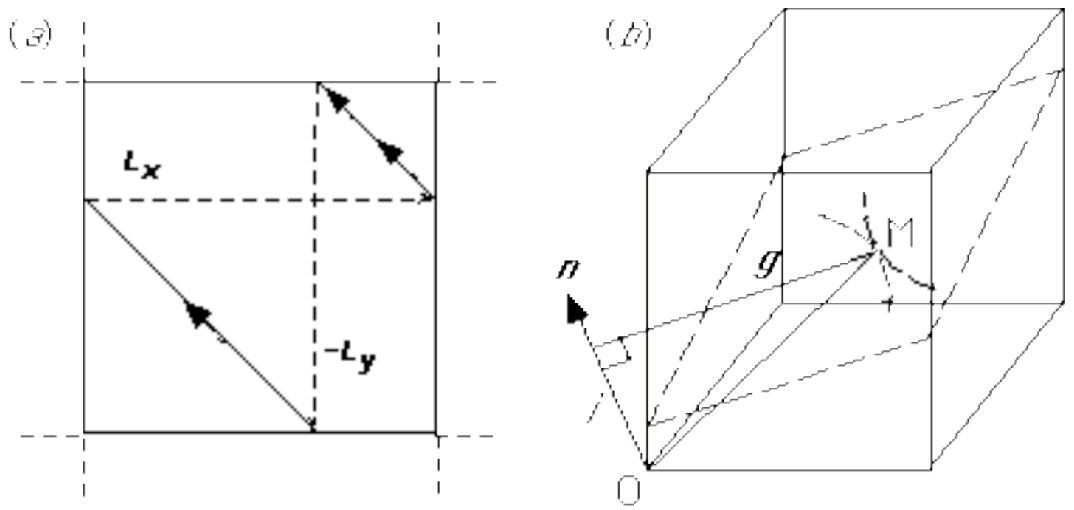

Figure 1. $a$ - In a square cell, a point moving along a direction parallel to the second diagonal annihilates with its image after two modulo translations. $b$ - Self-annihilation in threedimensions and in a slip plane of normal $\boldsymbol{n}$.

We now consider the images of a three-dimensional trajectory in the primary cell (cf. Fig. 1-b). In what follows, the primary simulation cell has $\{100\}$ faces and its dimensions are defined by the vectors $\left(\boldsymbol{L}_{\mathrm{x}}, \boldsymbol{L}_{\mathrm{y}}, \boldsymbol{L}_{\mathrm{z}}\right)$. The slip plane normal is denoted $\boldsymbol{n}(h, k, l)$, and the position of a point along an expanding dislocation loop is defined by the vector $\boldsymbol{O M}$, the arbitrary origin of the expansion, $\mathrm{O}$, being taken at the origin of the cell. The vector $\boldsymbol{O} \boldsymbol{M}$ can be decomposed as a sum of two components, one along the in the glide plane ( $g$ in Fig. 1-b) and the other along the slip plane normal. The latter allows distinguishing between slip planes of different heights in the simulation. For this purpose, we define a slip plane index, $i$, such that (Fig. 1-b):

$$
i=\boldsymbol{O M} . \boldsymbol{n}
$$

In each cell, this index goes from zero to a maximum value. Upon selfannihilation, the two interacting segments must have same slip plane index, as they are in the same slip plane. In the case depicted in Fig. 1- $b$, selfannihilation occurs between two images in a plane with non-zero index, which is not that of the loop expanding from the origin $(i=0)$. It can also occur in the plane $i=0$, between the loop and one of its images. These two situations will be discussed separately in section 3. Finally, we define the total translation, $\boldsymbol{T}$, as the sum of the whole set of modulo translations upon self-annihilation:

$$
\boldsymbol{T}=u \boldsymbol{L}_{\mathrm{x}}+v \boldsymbol{L}_{\mathrm{y}}+w \boldsymbol{L}_{\mathrm{z}}
$$


In this expression, $u, v$, and $w$ are integers counting the number of cell boundary crossings before self-annihilation. The total translation $\boldsymbol{T}$ has a glide component and a component along the normal to the slip plane. Thus, the condition for self-annihilation of a segment in a slip plane with index $i$ is simply written $\boldsymbol{T} . \boldsymbol{n}=i$, or, in developed form:

$$
h u L_{\mathrm{x}}+k v L_{\mathrm{x}}+l w L_{\mathrm{x}}=i
$$

In the same way, all the slip plane indexes met during the glide path $\boldsymbol{O M}$ are a linear combination of $h L_{\mathrm{x}}, k L_{\mathrm{y}}$ and $l L_{\mathrm{z}}$. The greatest common divisor of these three last quantities defines, along the normal $n$, a one-dimensional lattice that contains all the possible slip plane indexes available to dislocations. This property is, actually, the only one that is needed to apply the general solutions developed below to three-dimensional DD simulations. It also defines the smallest dipole height that can be met in a given slip system.

Before coming back to the general calculation of the self-annihilation distances, we examine particular slip geometries leading to very short annihilation distances.

\subsection{Direct self-annihilations}

In general, the modulo translations are not in a slip plane. This can, however, happen for particular slip geometries. As schematically depicted in Fig. 2-a, when a dislocation line propagates along a direction parallel to a modulo translation, self-annihilation occurs after one boundary crossing, irrespective of the cell shape, cubic or orthrhombic. This type of situation is not uncommon and is met typically with $\{100\}$ and $\{110\}$ slip planes in some simple cubic structures. For example, the $\{110\}$ slip planes in the bcc structure contain a modulo translation parallel to $<001>$.

A solution to this problem consists in shifting a set of parallel boundaries by a translation $\square_{\mathrm{ij}}$ (Fig. 2-a). The first index refers here to the boundary normal and the second one to the shift direction. In lattice-based simulations, the coherency of the slip planes across the interface has to be preserved and $\square_{\mathrm{ij}}$ is a translation of the simulation lattice. The shift vector must also preserve the continuity of the simulated material. For instance normal shifts $\square_{\mathrm{i}}$ are forbidden, as they introduce voids or matter overlaps. For the same reason, simultaneous symmetrical shears on different boundaries, like $\square_{\mathrm{j}}$ and $\square_{\mathrm{i}}$ are forbidden, and of the three non-zero shears, only two can be applied simultaneously. Thus, in three dimensions, the problem can be solved for two boundaries only. Another solution is, then, needed, for the case of $\{100\}$ slip, where direct self-annihilation can occur in three different directions.

An alternative solution consists in rotating the crystallographic orientations with respect to the primary cell (Fig. 2-b), in order to tilt the slip planes away from the direction of the modulo translation. In discretized 
simulations, the rotated lattice must be such that the summits of the unit cell have integer Miller indexes, in order to ensure crystallographic compatibility between the primary cell and its replicas. In cubic or orthorhombic structures, a convenient solution consists in rotating the crystallographic axes around simple crystallographic directions, for instance $<001>$. This method can be employed alone, as one rotation can be applied along each $<100>$ direction, at the expense of some complexity, or in combination with shifts. The optimization of the cell dimensions presented below can be carried out in the presence of shifts and rotations (cf. section 3.4).
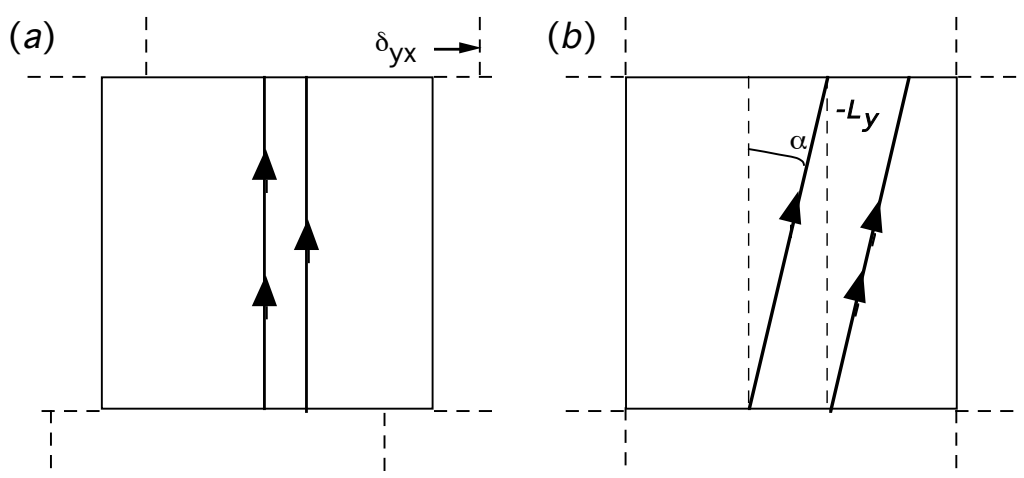

Figure 2. Two-dimensional sketches of direct self-annihilations after a single boundary crossing. $a-$ A vertical slip direction is transformed into itself after one boundary crossing (single arrow). The problem is solved by shifting the neighboring cells with respect to each other (here by $\square_{\mathrm{yx}}$ ) so that the glide path and its image (double arrow) do not coincide. $b-$ The same problem is solved by introducing a rotation $\square$ of the crystallographic axes with respect to the primary cell.

\section{CONTROLLING SELF-ANNIHILATIONS}

\subsection{Isotropic and Anisotropic Dislocation Loops}

In three-dimensions, the self-annihilation distance of a dislocation segment in a given slip plane depends on its propagation direction. Its minimum value, which is achieved for a particular direction, corresponds to the first self-annihilation occurring during a simulation. This quantity is the one that should be controlled for a proper use of PBCs. Two model situations are considered in what follows, which correspond to common configurations found in the practice. In fcc crystals, dislocation loops expand with rather isotropic shapes and mean-free paths of the order of a several tens of microns ${ }^{4}$. At low temperatures in bcc crystals and for prismatic slip in hep crystals, screw dislocations experience a strong lattice friction and 
non-screw segments are comparatively highly mobile. These segments can have long mean-free paths, perhaps up to one hundred of microns or more in pure crystals; their propagation results in the formation of loops with very anisotropic shapes ${ }^{5}$.

\subsection{Geometry in the Extended Slip Plane}

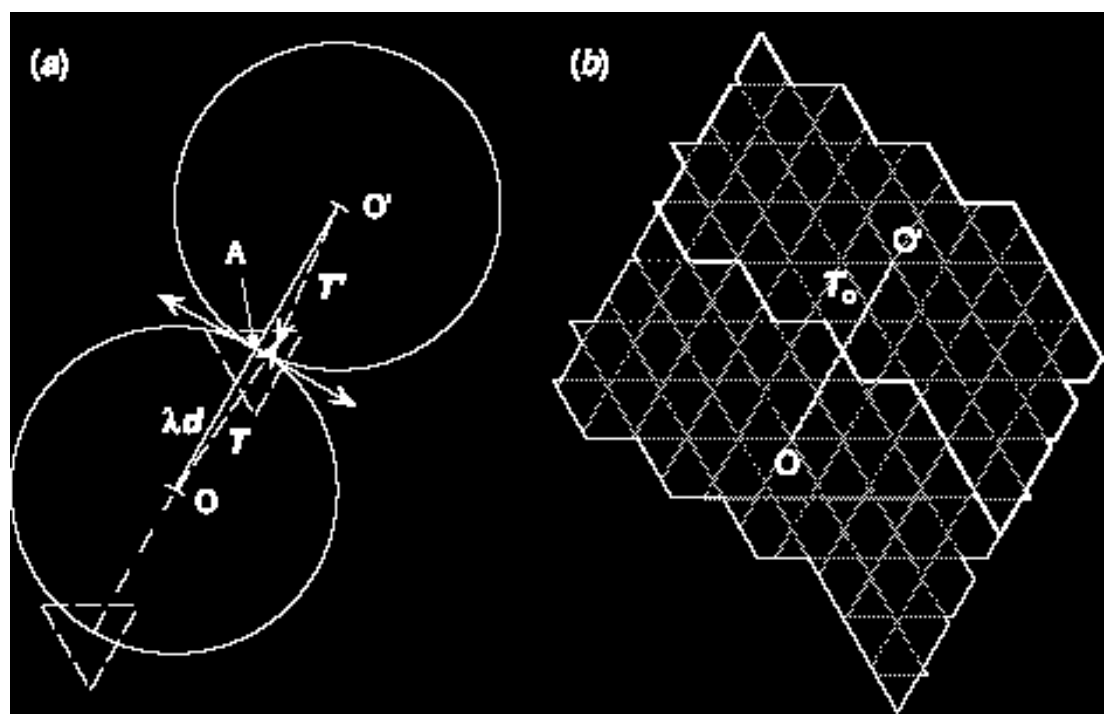

Figure 3. $a$ - A circular dislocation loop expands from the origin $\mathrm{O}$ in a slip plane of normal $\boldsymbol{n}$. Along a direction $\boldsymbol{d}$, and for a certain radius $\square$, the first self-annihilation occurs in A with an image that moved in the opposite direction (see text for other details). $b$ - Tiling of a (111) slip plane in the fcc structure by polygonal intersections with a periodic array of orthorhombic simulation cells. The latter have here dimensions proportional to the set of incommensurate numbers $(40,30,31)$. Two supercells are shown, of which the boundaries were computed from the condition that each supercell only contains polygons with different indexes.

Figure 3-a, schematically depicts the self-annihilation of an isotropic dislocation loop expanding by glide from the origin $\mathrm{O}$ of the primary cell (initial index $i_{\mathrm{o}}=0$ ). As indicated in section 2.1 the origin can be chosen in an arbitrary manner without loss of generality. The first self-annihilation event occurs along a direction $\boldsymbol{d}\left(d_{\mathrm{x}}, d_{\mathrm{y}}, d_{\mathrm{z}}\right)$, after a glide path or selfannihilation radius $\square$.

The intersections of a slip plane with the periodic array of simulation cells define a tiling of contiguous polygons with various indexes and shapes. This tiling is necessarily periodic since there is a finite number of possible indexes $i$, which corresponds to an equivalent number of distinct polygonal intersections. One can thus define in the slip plane a periodic array of supercells, of which the periodicity in any direction is defined by the periodicity of the indexes of the polygonal intersections. The supercells have complex shapes, as shown in the example given in Figure 3-b. This example 
also illustrates the problems met with non-optimal cell dimensions: the selfannihilation radius is rather small in this case (1.27 times a cell diagonal) and the supercell is rather anisotropic, which means that self-annihilation occurs along different directions in the various $\{111\}$ slip planes.

In Fig. 3- $a$, self-annihilation occurs when a portion of the expanding loop meets in the same polygonal intersection with an image propagating in the opposite direction. The two segments meet with a common tangent and a common normal parallel to the propagation direction $\boldsymbol{d}$. Thus, the first selfannihilation event on the dislocation loop occurs along the direction of the shortest distance between images of the origin in the superlattice, $\boldsymbol{T}_{\mathrm{o}}=\boldsymbol{O} \boldsymbol{O}^{\prime}$ (cf. Fig. 3-b). The self-annihilation diameter is then $2 \square=\boldsymbol{T}_{\mathrm{o}}$, and the selfannihilation radius, $\square$, is half this value (Fig. 3-a). As already mentioned, the two total translations $\boldsymbol{T}$ and $\boldsymbol{T}^{\prime}$ (dashed lines in Fig. 3-a) are not in the slip plane. Further, they do not necessarily correspond to symmetrical sequences of modulo translations. For instance, a portion of loop expanding in the primary cell starts with a glide sequence, whereas its images start by crossing cell boundaries, which implies one additional modulo translation.

The geometrical considerations developed in the previous sections are now used to calculate a relation between the self-annihilation radius and the cell dimensions. The practical applications are outlined in section 3.4.

\subsection{Self-Annihilation of Isotropic Loops}

The direction of first self-annihilation is that of the smallest vector connecting two neighboring supercells, $\boldsymbol{T}_{\mathrm{o}}$. Considering the total translations $\boldsymbol{T}$ and $\boldsymbol{T}^{\prime}$, we see from Fig. 3- $a$ that $\boldsymbol{T}_{\mathrm{o}}=\boldsymbol{T}-\boldsymbol{T}^{\prime}$ and, further, that $\boldsymbol{T}_{\mathrm{o}}=2 \square \boldsymbol{d}$, so that the condition for first self-annihilation is written:

$$
2 \square \boldsymbol{d}+\boldsymbol{T}_{\mathrm{o}}=0 .
$$

In projection along the slip plane normal, we have $\boldsymbol{T}_{\mathrm{o}} \boldsymbol{n}=0$, or:

$$
h u L_{\mathrm{x}}+k v L_{\mathrm{x}}+l w L_{\mathrm{x}}=0
$$

In this expression, the unknows are the numbers of boundary crossings $u, v$ and $w$. Linear equations, of which the solutions searched for are integer numbers, like Eq. (5), are known under the name of first-degree Diophantine equations (after Diophantes of Alexandria, cf. Bashmakova 1997); their solutions can be obtained with the help of various algorithms. The first selfannihilation corresponds to the set of integers $(u, v, w)$ leading to the translation vector between supercell origins of smallest magnitude, $\boldsymbol{T}_{\mathbf{0}}$. The self-annihilation diameter is then obtained from Eq. (4):

$$
2 \square=\sqrt{\left(u L_{\mathrm{x}}\right)^{2}+\left(v L_{\mathrm{y}}\right)^{2}+\left(w L_{\mathrm{z}}\right)^{2}}
$$




\subsection{Self-annihilation of Anisotropic Loops}

The anisotropic loops have in practice quasi-rectangular shapes and the fast moving portions, of small length, can be approximated by segments of infinitesimal length propagating along the direction $\pm \boldsymbol{d}$. In contrast with the previous case, the direction $\boldsymbol{d}$ is fixed by a physical argument and is not necessarily connecting the origins of neighboring supercells. Anisotropic loops or segments can, thus, cross several supercells before self-annihilating. As a consequence, their trajectories have one interesting property, which is not formally developed here by lack of space. Every time the boundary of a supercell is crossed by a small segment, an image of the latter appears in all the supercells through the operation of the PBCs. The successive images are shifted by a constant amount, due to the fact that the propagation direction is not, in general, parallel to direction connecting the origins of neighboring supercells. It follows that the images of the trajectories of anisotropic loops in a slip plane are equidistant. Then, a necessary condition for treating the short segments as infinitesimal ones is that their length be smaller than the spacing between their trajectories.

If an edge segment is emitted in only one direction, its return glide distance is $\square$. There is then no annihilation, but a spurious spatial correlation is introduced (to simplify notations, we still call $\square$ a self-annihilation radius). Each segment starts from the origin $(i=0)$ and self-interacts with an image at the origin of another supercell. Equation (3) of section 2.1, taken with $i=0$ applies to this situation. However, Eq. (3), which is also a first-order Diophantine equation, does not explicitly include the propagation direction $\boldsymbol{d}$ and yields solutions for all directions in a given slip plane. A more transparent solution can be derived as follows.

The position of the self-annihilation event in the extended plane is brought back into the primary cell by subtracting the total translation from the self-annihilation radius. Then (cf. section 2.1 and Fig. 1-b):

$$
\boldsymbol{O M}=\square d-T
$$

Upon self-annihilation at the origin, we have $\boldsymbol{O M}=0$ and Eq. (7) reduces to $\square \boldsymbol{d}-\boldsymbol{T}=0$, or, in developed form :

$$
\frac{\square}{\sqrt{d_{\mathrm{x}}^{2}+d_{\mathrm{y}}^{2}+d_{\mathrm{z}}^{2}}}=\frac{u L_{\mathrm{x}}}{d_{\mathrm{x}}}=\frac{v L_{\mathrm{y}}}{d_{\mathrm{y}}}=\frac{w L_{\mathrm{z}}}{d_{\mathrm{z}}},
$$

where the $d_{i}$ are integer crystallographic indexes. This equation reduces to Eq. (3) by introducing the condition $\boldsymbol{d} . \boldsymbol{n}=0$, which expresses that the direction $\boldsymbol{d}$ is in the slip plane of normal $\boldsymbol{n}$. The set of integer numbers $(u, v$, $w$ ) is obtained from the three equations at the right-hand side of Eq. (8): 


$$
v=u \frac{L_{\mathrm{x}} d_{\mathrm{y}}}{L_{\mathrm{y}} d_{\mathrm{x}}} ; \quad w=u \frac{L_{\mathrm{x}} d_{\mathrm{z}}}{L_{\mathrm{z}} d_{\mathrm{x}}} .
$$

This equation has an infinite number of solutions. The first selfannihilation is obtained through the additional condition that it corresponds to the total translation vector $\boldsymbol{T}$ of smallest magnitude, i.e., to the smallest distance between cell origins. The self-annihilation radius is then determined from the left-hand side of Eq. (8).

\subsection{Implementation}

In practice, several conditions are initially prescribed. The average dimension of the primary cell should be large with respect to the typical length scale of interest, for instance an average distance between dislocations or the diameter of a dislocation cell. This leads to average dimensions of typically $10 \square \mathrm{m}$ for standard mass simulations. The value of the selfannihilation radius is then prescribed according to the physical situation considered. The orthorhombic cell should not be too much distorted with respect to the cubic shape, so that all crystallographically equivalent active slip planes have equal weight in the simulation. The cell dimensions should then be proportional to a set of three incommensurate numbers centered around an average value.

In the anisotropic case, several possible solutions can be obtained directly from Eqs. (8) and (9). In the isotropic case, The coupled equations (5) and (6) are solved numerically, which also leads to several possible solutions. The condition that the supercells have isotropic shapes ensures that all equivalent slip planes of a same family have comparable self-annihilation radii. It is used to select the optimal solution.

Several situations related to self-annihilation can also be treated via firstorder Diophantine equations. This includes, in particular, the formation of dipoles with small heights, which can be troublesome during model simulations with small initial dislocation densities. The condition for selfannihilation is then replaced by the condition that self-interaction occurs in a cell where the interacting segments have nearest-neighboring indexes.

Finally, these solutions can easily be extended to the particular slip geometries where one has to use shifts and rotations to avoid premature selfannihilation (section 2.2). Rotations of the crystallographic lattice are cared of by carrying out calculations in the rotated lattice. Shifts applied to the boundaries are accounted for by replacing the modulo translations $L_{\mathrm{i}}$ by translations $\left(\boldsymbol{L}_{\mathrm{i}}+Z_{\mathrm{j}},\right)$ incorporating the shift on the face $\mathrm{i}$. 


\section{CONCLUDING REMARKS}

The self-annihilation of dislocation lines caused by the use of PBCs is an artifact that can be turned into an advantage. Indeed, the methods outlined in the present work allow setting the values of the self-annihilation distances and, in the same time, making sure that the effective dislocation mean-free paths are conform to physical expectations. This last length scale has a strong influence on the development of dislocation microstructures and its control allows drawing quantitative estimates from DD simulations ${ }^{3,7}$.

The two situations considered here, those of isotropic and anisotropic loops cover most of the cases of practical interest. To avoid severe artifacts occurring with particular slip geometries, two methods are proposed that consist in modifying the periodic arrangement of the simulation cells. These methods can be implemented in any DD simulation, discretized or not. The self-interaction distances of dislocations are prescribed through a choice of the dimensions of an orthorhombic simulation cell based on a quantitative analysis. To be implemented, the latter only involves the requirement that the slip planes available to the moving dislocations be located on a set of equidistant heights in the simulation cell.

\section{ACKNOWLEDGEMENTS}

The authors gratefully thank Pr. Robert Rudd (Lawrence Livermore National Laboratory) for sharing his views on the question of PBCs. Part of this work was conducted by one of the authors (RM) at the Centre Européen de Calcul Atomique et Moléculaire (CECAM) during a post-doctoral stay.

\section{REFERENCES}

1. Bulatov VV, Rhee M, Cai W., in Multiscale Modeling of Materials 2000, LP Kubin, R. Selinger, JL Bassani J.L. and K Cho eds, Warrendale, PA: Materials Research Society 2000, Symposium Proceedings Vol. 653, p. z1-3.

2. Schwarz K.W. 1988, Phys. Rev. B 85:108.

3. Madec R., Devincre B., Kubin L.P. 2002, Phys. Rev. Lett. 89:255508.

4. Ambrosi P., Shwink Ch.. 1978, Scripta Metall. 12:303.

5. Tang M., Kubin, L.P., Canova, G.R. 1998, Acta Mater. 46:9.

6. Bashmakova, I.G., Diophantes and Diophantine Equations. Washington DC: Math. Assoc. Amer.

7. Madec R., Devincre, B., Kubin, L.P. 2002, Scripta Mater. 47:689. 